\title{
Being in a position to know and closure
}

\author{
Jan Heylen
}

\begin{abstract}
The focus of this article is the question whether the notion of being in a position to know is closed under modus ponens. The question is answered negatively.

Keywords: knowledge; being in a position to know; having the possibility to know; having the capacity to know; closure.
\end{abstract}

Since the work by Cohen (1999), Williamson (2000) and Hawthorne (2004) the notion of being in a position to know has gained prominence. For instance, Schaffer (2007) makes extensive use of the notion to formulate what he takes to be the best invariantist, contextualist and contrastivist closure principles. For example, the best invariantist closure scheme is supposed to be the following: if an agent $s$ knows that $p_{1}$ and if $p_{1}$ entails that $p_{2}$, then $s$ is in a position to know that $p_{2}$ (Schaffer, 2007, p. 235). When glossing those closure principles he talks about the closure of the notion of being in a position to know under entailment. But that gloss is inaccurate: the conclusion is formulated with the help of the notion of being in a position to know, whereas the premiss is formulated with the help of the notion of knowledge.

The question I would like to address is whether or not being in a position to know is closed under modus ponens, where both the conclusion and the premisses are formulated with the help of the notion of being in a position to know. Let $K$ express 'the agent is in a position to know that'. Then the question is whether the following is valid:

(C) $K \phi, K(\phi \rightarrow \psi) \vdash K \psi$,

with the proviso that the epistemic agent knows the rule of modus ponens and knows how to apply it. Rosenkranz (2007, Section 4) explicitly endorses, defends and applies (C). Greenough (2009, Sections 6 and 8) also applies (C), so he is at least implicitly committed to it. I, on the other hand, reject (C).

First, note that it follows from (C) that being in a position to know is closed under the introduction rule for conjunction:

$$
K \phi, K \psi \vdash K(\phi \wedge \psi),
$$

given the assumption that one is in a position to know that $\phi \rightarrow(\psi \rightarrow(\phi \wedge \psi))$, which is an easy tautology. Suppose that $K(\phi \rightarrow(\psi \rightarrow(\phi \wedge \psi)))$. It follows by (C) that $(K \phi \rightarrow K(\psi \rightarrow(\phi \wedge \psi)))$. Given (C) it is a consequence of $K(\psi \rightarrow(\phi \wedge \psi))$ that $K \psi \rightarrow K(\phi \wedge \psi)$. It is a tautological consequence of the supposition that:

$$
(K \phi \wedge K \psi) \rightarrow K(\phi \wedge \psi)
$$


If the supposition is a theorem, then (1). Rosenkranz (2007, p. 78, 85) explicitly endorses and uses (1).

Second, if one is in a position to know that $\phi$, then it is possible for one to know that $\phi$. Let $\mathbf{K}$ express 'the agent knows that'. Then one can express the assumption as follows:

$$
K \phi \rightarrow \diamond \mathbf{K} \phi
$$

It follows from the cursory remarks made by Williamson (2000, p. 95):

To be in a position to know $p$, it is neither necessary to know $p$ nor sufficient to be physically and psychologically capable of knowing $p$. No obstacle must block one's path to knowing $p$. If one is in a position to know $p$, and one has done what one is in a position to do to decide whether $p$ is true, then one does know $p$. The fact is open to one's view, unhidden, even if one does not yet see it.

Based on the above quote Rosenkranz (2007, p. 69) writes the following:

We may accordingly lay down three necessary conditions for one's being in a position to know $p$ :

[(i) ] $p$ is true

[(ii) ] One is physically and psychologically capable of knowing $p$

[(iii) ] Nothing stands in one's way of successfully exercising these capabilities

Clearly, if one is in a position to know that $\phi$ only if one is physically and psychologically capable of knowing that $\phi$ and nothing stands in one's way of successfully exercising these capabilities, then being in a position to know $\phi$ is conditional on possibly knowing $\phi$. For my purposes, the possibility mentioned in (2) may be restricted to possibility relative to one's physical and psychological capabilities.

Rosenkranz (2007, p. 90, fn. 14) explicitly endorses a stronger connection between being in a position to know and possibly knowing:

So if it is possible for us to be in a position to know $p$ ( $\diamond K p$ ' holds), then it is also possible for us to know $p$ (' $\diamond$ ( $p$ is known to us)' holds), and conversely.

For our purposes it is not necessary to use the above stronger principle.

Not everyone may be willing to accept (2). Take Smithies (2012), who distinguishes between the notion of an epistemic position to know on the one hand and the notion of a doxastic position to know on the other hand. One may be merely in an epistemic position to know by, say, having knowledge-level justification, without being in a doxastic position to know, say, because one is unable to to form a belief based on the justification one possesses. With a reading of the possibility operator as possibility restricted to one's physical and psychological capabilities, being in an epistemic position to know does not entail possibly knowledge. Yet, there is still reason to accept that, if one is in a doxastic position to know, one possibly knows. In what follows I will take care to make my argument palatable for those who are on board with Smithies (2012). 
Third, assume that knowledge is closed under the elimination rule for conjunction:

$$
\mathbf{K}(\phi \wedge \psi) \vdash(\mathbf{K} \phi \wedge \mathbf{K} \psi) .
$$

This assumption is also very reasonable (Williamson, 2000, Sect. 12.2). Note that (a special case of) the deduction theorem (Hughes and Cresswell, 1996, p. 211) says that:

$$
\phi \vdash \psi \Leftrightarrow \vdash \phi \rightarrow \psi .
$$

In addition, note that the following is a rule in any normal modal logic (Hughes and Cresswell, 1996, p. 35, DR3):

$$
\vdash \phi \rightarrow \psi \quad \Rightarrow \quad \vdash \diamond \phi \rightarrow \diamond \psi .
$$

Given the above, it follows that:

$$
\diamond \mathbf{K}(\phi \wedge \psi) \vdash \diamond(\mathbf{K} \phi \wedge \mathbf{K} \psi) .
$$

Fourth, assume that, if one knows something, then one is in a position to know it:

$$
\mathbf{K} \phi \rightarrow K \phi .
$$

Whether the position in question is an epistemic one or a doxastic one should not matter.

Now everything is in place to argue against (1) and, therefore, indirectly against (C). Suppose that one is in a position to know that $\phi$ and that one is in a position to know that one does not know that $\phi$ :

$$
K \phi \wedge K \neg \mathbf{K} \phi .
$$

It follows by (1) that one is in a position to know that $\phi$ and that one does not know that $\phi$ :

$$
K(\phi \wedge \neg \mathbf{K} \phi) .
$$

By (2), it follows that it is possible to know that $\phi$ and that one does not know that $\phi$ :

$$
\diamond \mathbf{K}(\phi \wedge \neg \mathbf{K} \phi) .
$$

By (3), it follows that it is possible that one knows that $\phi$ and that one knows that one does not know that $\phi$ :

$$
\diamond(\mathbf{K} \phi \wedge \mathbf{K} \neg \mathbf{K} \phi) .
$$

Since $\mathbf{K}$ is factive, it follows that it is possible that one knows that $\phi$ and that one does not know that $\phi$ :

$$
\diamond(\mathbf{K} \phi \wedge \neg \mathbf{K} \phi) .
$$


Contradictions are impossible. ${ }^{1}$ Consequently, it is not the case that one is in a position to know that $\phi$ and that one is in a position to know that one does know that $\phi$ :

$$
\neg(K \phi \wedge K \neg \mathbf{K} \phi) .
$$

The conclusion then is that, if one is in a position to know that one does not know that $\phi$, then one is not in a position to know that $\phi(K \neg \mathbf{K} \phi \rightarrow \neg K \phi){ }^{2}$

The derived consequence is absurd. To build on an example from Williamson (2000, p. 272): suppose that my office contains an even number of books ( $p)$, but I don't know it $(\neg \mathbf{K} p)$, because I have never counted them, and I know it $(\mathbf{K} \neg \mathbf{K} p)$. Given (4), I am in a position to know that I don't know that the number of books in my office at the time of writing is even $(K \neg \mathbf{K} p)$. Yet, I am clearly in a position to know whether the number of books in my office at the time of writing is even $(K p)$, since I could just get up, walk to the book shelves and count them. And I would believe the result of the count. ${ }^{3}$

Principle (C) and its derivative, (1), are invalid. Even if Schaffer (2007) is right in claiming that the notion of being in a position to know figures in our best formulations of the closure principles, the notion itself is not closed under logical inference in general. This has consequences for the applications of $(C)$ in the literature, especially the applications found in (Rosenkranz, 2007) and (Greenough, 2009). In both articles a key role of principle (C) lies in the justification of two other principles. The argument starts from the assumption that we are in a position to know that we are not in a position to know falsehoods:

$$
\begin{gathered}
K(\phi \rightarrow \neg K \neg \phi) ; \\
K(\neg \phi \rightarrow \neg K \phi) .
\end{gathered}
$$

The application of (C) to the above yields:

$$
\begin{gathered}
K \phi \rightarrow K \neg K \neg \phi ; \\
K \neg \phi \rightarrow K \neg K \phi .
\end{gathered}
$$

\footnotetext{
${ }^{1}$ The inference from $\diamond \mathbf{K}(\phi \wedge \neg \mathbf{K} \phi)$ to a contradiction yields 'the independent result', viz. $\neg \backslash \mathbf{K}(\phi \wedge \neg \mathbf{K} \phi)$, that is used in the paradox of knowability, which is a derivation of omniscience, i.e. $\forall p(p \rightarrow \mathbf{K} p)$, from the knowability principle, i.e. $\forall p(p \rightarrow \diamond \mathbf{K} p)$ - see (Fitch, 1963; Brogaard and Salerno, 2013). Although the paradox of knowability has generated a lot of discussion, most of the paradox-avoiding strategies focus on syntactic or semantic restrictions of the knowability principle, while few of the options on the table threaten the 'independent result', with the exceptions of (i) denials of (i-a) the closure of knowledge under the elimination rule for conjunction and (i-b) the factivity of knowledge and (ii) the adoption of a paraconsistent background logic. Option (i-b) is not going to be an attractive one for Rosenkranz (2007) and Greenough (2009), because they are committed to the claim that one is in a position to know that one does not know falsehoods, as we will see later. I will leave contemplation of the other options to the reader.

${ }^{2}$ The argument and its conclusion are similar but not identical to (Heylen, 2015, Lemma 3.2, Theorem 3.3). The differences have to do with the fact that the latter is exclusively concerned with the notion of a priori knowability, understood as the possibility of having a priori knowledge $\left(\diamond \mathbf{K}_{a}\right)$. Therefore, there is no need for assumptions (2) and (4). Moreover, the conclusion is not (epistemically) mixed: if it is a priori knowable that one does not a priori knows some truth, then that truth is not a priori knowable $\left(\diamond \mathbf{K}_{a} \neg \mathbf{K}_{a} \phi \rightarrow \neg \diamond \mathbf{K}_{a} \phi\right)$.

${ }^{3}$ The objection raised against $K \neg \mathbf{K} \phi \rightarrow \neg K \phi$ differs from the the so-called 'Socratic objection' raised against $\diamond \mathbf{K}_{a} \neg \mathbf{K}_{a} \phi \rightarrow \neg \diamond \mathbf{K}_{a} \phi$ in (Heylen, 2015, p. 367), because the latter involves only a priori knowledge, whereas the example here involves empirical knowledge.
} 
Rosenkranz (2007, p. 78) uses the contrapositions of (7) and (8), while Greenough (2009, p. 406) uses versions of (7) and (8) in which knowledge is indexed to a knowledge basis. In any case, in the light of the invalidity of (C) an important challenge is to investigate whether and how (7) and (8) can still be justified.

Acknowledgements. The author wishes to thank two anonymous reviewers and members of the Leuven Epistemology Group for useful feedback.

\section{References}

Brogaard, B. and J. Salerno (2013). Fitch's paradox of knowability. In E. N. Zalta (Ed.), The Stanford Encyclopedia of Philosophy (Winter 2013 ed.).

Cohen, S. (1999). Contextualism, Skepticism, and the Structure of Reasons. Philosophical Perspectives 13(s13), 57-89.

Fitch, F. B. (1963). A logical analysis of some value concepts. Journal of Symbolic Logic 28(2), $135-142$.

Greenough, P. (2009). On What It is to Be in a Quandary. Synthese 171(3), 399-408.

Hawthorne, J. (2004). Knowledge and Lotteries. Oxford University Press.

Heylen, J. (2015). Closure of a priori knowability under a priori knowable material implication. Erkenntnis 80(2), 359-380.

Hughes, G. E. and M. J. Cresswell (1996). A New Introduction to Modal Logic. London: Routledge.

Rosenkranz, S. (2007). Agnosticism as a third stance. Mind 116(461), 55-104.

Schaffer, J. (2007). Closure, Contrast, and Answer. Philosophical Studies 133(2), 233-255.

Smithies, D. (2012). Mentalism and Epistemic Transparency. Australasian Journal of Philosophy 90(4), 723-741.

Williamson, T. (2000). Knowledge and its limits. New York: Oxford University Press. 